\title{
Simulation of a Wall-Bounded Flow using a Hybrid LES/RAS Approach with Turbulence Recycling
}

\author{
Jesse R. Quinlan ${ }^{1}$, James C. McDaniel ${ }^{2}$ \\ University of Virginia, Charlottesville, VA, 22903 \\ and \\ Robert A. Baurle ${ }^{3}$ \\ NASA Langley Research Center, Hampton, VA, 23666
}

Simulations of a supersonic recessed-cavity flow are performed using a hybrid largeeddy/Reynolds-averaged simulation approach utilizing an inflow turbulence recycling procedure and hybridized inviscid flux scheme. Calorically perfect air enters the threedimensional domain at a free stream Mach number of 2.92. Simulations are performed to assess grid sensitivity of the solution, efficacy of the turbulence recycling, and effect of the shock sensor used with the hybridized inviscid flux scheme. Analysis of the turbulent boundary layer upstream of the rearward-facing step for each case indicates excellent agreement with theoretical predictions. Mean velocity and pressure results are compared to Reynolds-averaged simulations and experimental data for each case, and these comparisons indicate good agreement on the finest grid. Simulations are repeated on a coarsened grid, and results indicate strong grid density sensitivity. The effect of turbulence recycling on the solution is illustrated by performing coarse grid simulations with and without inflow turbulence recycling. Two shock sensors, one of Ducros and one of Larsson, are assessed for use with the hybridized inviscid flux reconstruction scheme.

\section{Nomenclature}

$\begin{array}{ll}C_{K l e b} & =\text { Klebanoff function constant } \\ C_{\mu} & =\text { turbulence model constant } \\ D & =\text { distance to nearest solid surface } \\ d & =\text { cell interface flux } \\ F_{i+1 / 2} & =\text { von Kármán constant } \\ \kappa & =\text { turbulence kinetic energy } \\ k & =\text { shock sensor constant } \mathrm{i} \\ L_{s s, i} & =\text { pressure } \\ P & =\text { primitive variable vector } \\ q & =\text { time } \\ t & =\text { temperature } \\ T & =\text { streamwise velocity component } \\ u & =\text { transverse velocity component } \\ v & =\text { constant controlling the LES/RAS blending }\end{array}$

\footnotetext{
${ }^{1}$ Graduate Student, Department of Mechanical and Aerospace Engineering, 122 Engineer's Way, and AIAA student member.

${ }^{2}$ Professor, Department of Mechanical and Aerospace Engineering, 122 Engineer's Way, and AIAA Fellow.

${ }^{3}$ Aerospace Engineer, Hypersonic Airbreathing Propulsion Branch, 12 Langley Blvd, and AIAA Associate Fellow.
} 


$\begin{array}{lll}\delta_{i j} & =\text { Kronecker delta function } \\ \delta & = & \text { boundary layer thickness } \\ \rho & = & \text { density } \\ \Gamma & = & \text { LES/RAS blending function } \\ \Gamma_{\mathrm{SS}} & = & \text { shock sensor coefficient } \\ v & = & \text { kinematic molecular viscosity } \\ \omega & = & \text { turbulence frequency } \\ \chi & = & \text { Taylor micro-scale } \\ \text { inf } & = & \text { free-stream value } \\ i, j, k & = & \text { spatial coordinates } \\ K l e b & = & \text { Klebanoff } \\ r e s & = & \text { resolved } \\ S G S & = & \text { subgrid-scale } \\ H Y & = & \text { hybrid } \\ S R & = & \text { symmetric reconstruction } \\ U P & = & \text { upwind } \\ & = & \text { fluctuation } \\ * & = & \text { normal to a surface } \\ + & = & \text { wall unit }\end{array}$

\section{Introduction}

In order to address some of the fundamental limitations of Reynolds-averaged simulation (RAS) approaches while also exploiting the strengths of large-eddy simulation (LES) approaches, researchers are more frequently using a blended LES/RAS approach. This is especially true for use with high Reynolds number supersonic wall-bounded flows, which exhibit inherent unsteadiness, turbulent shock-boundary layer interactions, and other complex flow phenomena. Whereas the RAS equations require modeling of turbulence at all length scales, the LES equations only require modeling of turbulent motions of a length scale smaller than a characteristic filter width; the largest turbulent motions are resolved naturally through the solution of the filtered governing equations. Since these larger turbulent structures are responsible for the majority of mass, momentum, and energy transport, LES simulations are wellsuited for high Reynolds number flows. Unfortunately, the grid densities required for pure LES simulations of wallbounded flows often render the approach computationally infeasible for problems of practical importance.

In this study, a hybrid LES/RAS approach is used in conjunction with a turbulence recycling procedure to simulate the Settles, et. al. ${ }^{1,2,3}$ supersonic flow over a rearward facing step and subsequent reattachment along an inclined wall. The recycling process allows for the introduction of resolved turbulent content in an equilibrium turbulent boundary layer without having to simulate the entire development of the boundary layer. The chosen simulation framework is assessed in its ability to predict mean flow properties within the approach turbulent boundary layer, through the detached shear layer, and along the inclined wall. Assessments of the LES/RAS simulations are made using steady-state RAS results and experimental data.

This study serves a dual-purpose; in addition to validating the application and extension of the hybrid LES/RAS methodology, these simulations assess several recent developments to the Viscous Upwind aLgorithm for Complex flow ANalysis (VULCAN) computational fluid dynamics (CFD) code. ${ }^{4}$ These recent developments include reduced-dissipation numerics, a hybridized flux reconstruction scheme, and inflow turbulence recycling. Specific objectives of the current study include: assessment of the applicability of the hybrid LES/RAS method for cavity flows, isolation of the effect of inflow turbulence recycling on the flowfield statistics, determination of any grid sensitivity of the solution, and evaluation of shock sensor choice for use with the hybridized inviscid flux reconstruction scheme. These objectives are achieved through the study of six distinct cases that are parameterized by solution method, inflow treatment, grid size, and shock sensor and are summarized in Table 1. Elsewhere in this paper, each of the simulation cases will be referenced using the case ID stated in Table 1. For example, references will be made using "case R35D", rather than "case one", where R, 35, and D indicate recycling, 35 million grid cells, and Ducros sensor, respectively. 
Table 1. Simulation cases parameterized by inflow treatment, grid size, and shock sensor.

\begin{tabular}{c|c|c|c|c|c} 
Case & Case ID & Solution Method & Inflow Treatment & Grid Cells & Shock Sensor \\
\hline 1 & R35D & LES/RAS & Recycling & $35 \mathrm{e} 6$ & Ducros \\
\hline 2 & RR8D & LES/RAS & Recycling & $8.8 \mathrm{e} 6$ & Ducros \\
\hline 3 & NR8D & LES/RAS & No recycling & $8.8 \mathrm{e} 6$ & Ducros \\
\hline 4 & R35L & LES/RAS & Recycling & $35 \mathrm{e} 6$ & Larsson \\
\hline 5 & R8L & LES/RAS & Recycling & $8.8 \mathrm{e} 6$ & Larsson \\
\hline 6 & NR8RAS & RAS & No recycling & $8.8 \mathrm{e} 6$ & None
\end{tabular}

In the following section, the original wind-tunnel experiment and major flow features are described. In section three, the hybrid LES/RAS formulation and associated turbulence closure models are described. Details of the numerical formulation are included in section four. After describing the grids and computational executions in section five, the results and accompanying discussion are presented in section six. Conclusions and summarizing comments are included in the final section.

\section{Physical Flow}

The flow chosen for the current investigation was first studied by Settles, et. al. in the early 1980s. ${ }^{1,2,3}$ The original experiment was performed in the Princeton University $20 \mathrm{~cm}$ by $20 \mathrm{~cm}$ High Reynolds Number Supersonic Wind Tunnel and consisted of Mach 2.92 air entering the tunnel test section at a stagnation temperature of $258 \mathrm{~K}$, a stagnation pressure of $0.69 \mathrm{MPa}$, and a free stream unit Reynolds number of $6.7 \times 10^{7} / \mathrm{m}$. The test article, presented in Fig. 1, was designed such that a turbulent boundary layer developed along a flat plate before separating over a sharp corner. The free shear layer formed over the subsequent cavity and reattached along a wall of length $18 \mathrm{~cm}$ inclined at $20^{\circ}$. The position of the inclined wall was adjusted before taking measurements until no change in pressure or flow direction occurred due to the separating boundary layer. This procedure was necessary due to the high sensitivity of the shear layer location to the pressure through the test region. The cavity depth was $2.54 \mathrm{~cm}$, and the model was unaffected by the tunnel sidewall boundary layers due to its positioning away from the walls. In order to further promote two-dimensionality, aerodynamic fences lined the edges of the inclined wall. Mean flow measurements were made using a traversing set of pitot and static pressure probes, and these measurements, in conjunction with an assumption of constant total temperature, enabled the researchers to calculate Mach number, velocity, density, and other properties. A spark shadowgraph enabled observation of major flow features.

The original experiment was designed to illustrate the complex fluid phenomena comprising the reattachment of a free shear layer in a supersonic flow. The effect of turbulence on the flowfield is prominent, as documented in the original review of the experiment and in several subsequent numerical investigations. ${ }^{2,5}$ Major flow features observed in the experiment are illustrated in Fig. 2. After the approach boundary layer detaches at the step corner, a free shear layer forms over the cavity. As the shear layer reattaches at the inclined wall, fluid is entrained into the cavity, thereby forming a recirculation zone. The inherent unsteadiness of the shear layer alters the location of reattachment and subsequently affects the fluid entrainment. Together, the shear layer unsteadiness, altered attachment point, and recirculation zone form a feedback loop that gives rise to a low-frequency flapping motion of the shear layer. Furthermore, as the shear layer compresses and reattaches, an oblique compression shock is formed off the inclined wall.

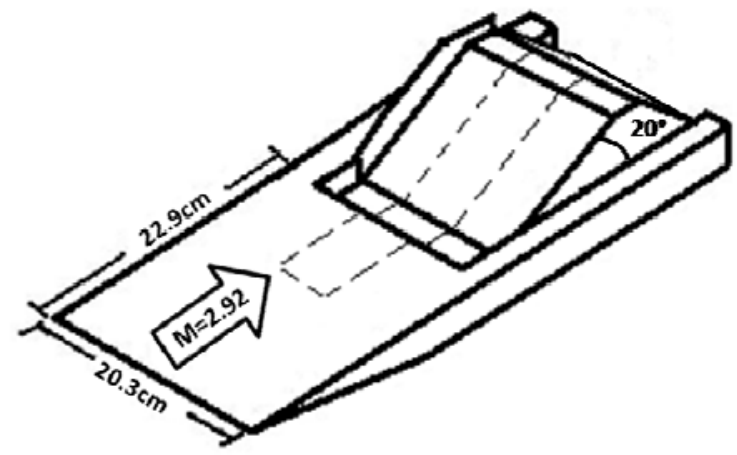

Figure 1. Experimental setup with one aerodynamic fence removed and the computational domain footprint signified by dashed lines. 


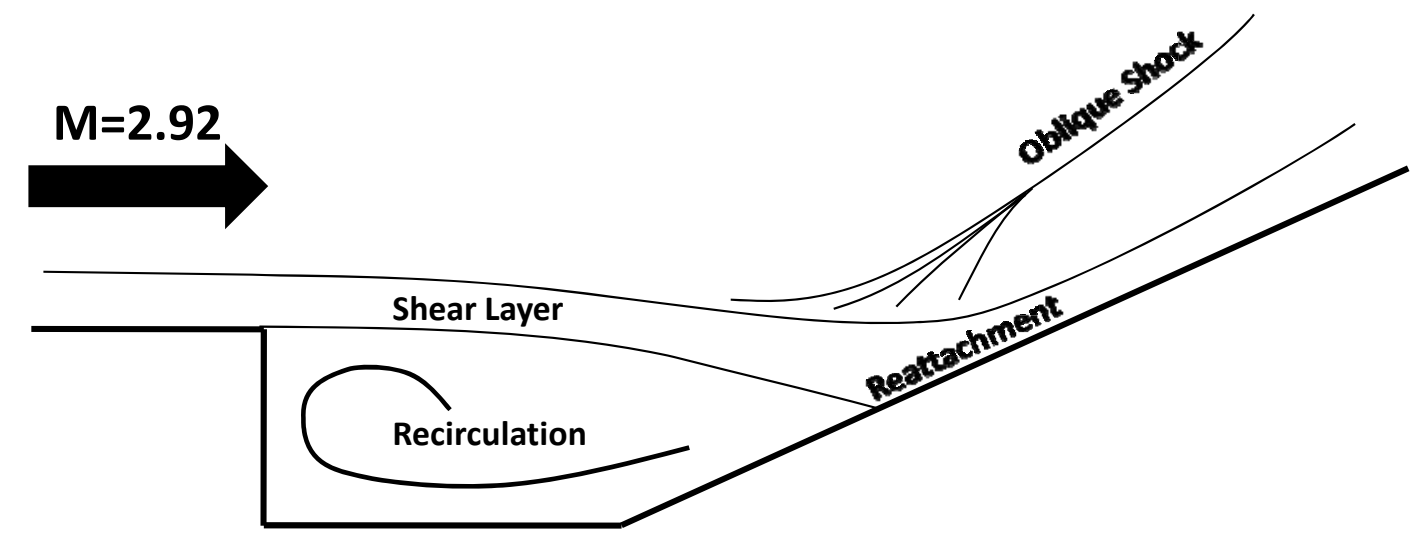

Figure 2. Major flow features observed during the experiment.

\section{Hybrid LES/RAS Formulation}

In the current hybrid LES/RAS approach, blending of the solution between LES and RAS throughout the physical domain is achieved via blending of the closure models. The current implementation in VULCAN is designed such that the choice of RAS and SGS closure models is delegated to the user, thereby allowing significant flexibility. An overview of the detailed description of this formulation found in Ref. 6 is presented here.

VULCAN solves the Navier-Stokes equations using a cell-centered finite-volume scheme and an upwind formulation for calorically or thermally perfect gases. Various one-equation and two-equation turbulence models are available for treating the turbulence closure. The code has been used extensively for simulating high-speed reacting flows in its RAS capacity with much success. However, by its nature, the RAS formulation is inadequate for the study of strongly unsteady flows, and the turbulence models used to close the RAS equations are often questionable for complex flowfields of engineering interest. The current hybrid LES/RAS capability enables VULCAN to be used more reliably in highly unsteady applications, in which large-scale turbulence and transients play an important role in simulating and understanding the evolution of a flowfield.

The blending of the LES and RAS approaches is accomplished using a flow- and grid-dependent blending of the chosen turbulence closure models. In this work, the Menter-BSL turbulence model ${ }^{7}$ is used to close the RAS equations. This model requires the solution of two additional equations - a transport equation for turbulent kinetic energy and a transport equation for turbulence frequency. To model the subgrid-scale (SGS) turbulence for use in LES regions, the one-equation model of Yoshizawa and Horiuti ${ }^{8}$ is used. This model requires the solution of a transport equation for the SGS turbulence kinetic energy (TKE). A detailed description of the turbulence models and the complete set of hybridized governing equations are found in Ref. 5.

The LES/RAS blending function is designed such that the RAS formulation transitions to the LES formulation in the outer region of the boundary layer, near the upper edge of the log layer. As previous studies have indicated ${ }^{9}$, targeting the transition for this region at the edge of the log layer within the turbulent boundary layer yields the most resolved turbulence without adversely affecting the universality of the boundary layer. The blending from LES to RAS is done smoothly using the following function ${ }^{10}$ :

$$
\Gamma=\frac{1}{2}\left(1-\tanh \left[5\left(\frac{\kappa}{\sqrt{C_{\mu}}} \eta^{2}-1\right)-\phi\right]\right), \quad \eta=\frac{d}{\alpha \chi}
$$

where the Taylor microscale is defined as:

$$
\chi=\sqrt{v / C_{\mu} \omega}
$$

The blending function input parameter, $\alpha$, is set by the user such that $\Gamma=0.99$ at the targeted average LES/RAS transition point, and is set to $\operatorname{atanh}(0.98)$ to fix the balancing position, where $\kappa \eta^{2}=\sqrt{C_{\mu}}$, to $\Gamma=0.99$. Performing the procedure outlined in Ref. 11 for the chosen flow yields a value of $\alpha=32.8$ for the turbulent approach boundary 
layer, which forces the switch from RAS to LES at the outer edge of the log layer (corresponding to a $d^{+}$of about 1080).

\section{Numerical Formulation}

The simulations performed for this investigation are an early application of several recent numerical developments to the VULCAN CFD code. VULCAN was originally designed to serve as a robust, upwind, RAS solver for application to high-speed reacting and non-reacting flows. In recent years, VULCAN has been expanded to include LES and hybrid LES/RAS capabilities, which drove an investment in various higher-order numerics. Several of these methods are used in the current study and are described in detail below.

\section{A. Low-Dissipation Numerics and Hybridized Inviscid Flux Scheme}

In order to reduce numerical dissipation in smooth flow regions, while maintaining the dissipation necessary to capture discontinuities elsewhere, a hybridized flux scheme is used to reconstruct interface inviscid fluxes. This hybridization is implemented as the linear combination of a low-dissipation, symmetrically-reconstructed inviscid flux and a conventional upwind-biased formulation. The low-dissipation flux formulation is described by Gieseking et al., ${ }^{12}$ in which the left and right states are reconstructed to higher-order accuracy at the cell interfaces using a fourpoint symmetric interpolation on the primitive variables of the form:

$$
\vec{q}_{L, i+\frac{1}{2}}^{S R}=\vec{q}_{R, i+\frac{1}{2}}^{S R}=\frac{7}{12}\left(\vec{q}_{i}+\vec{q}_{i+1}\right)-\frac{1}{12}\left(\vec{q}_{i+2}-\vec{q}_{i-1}\right)
$$

The low-dissipation interface flux is computed using a conventional average of the left and right fluxes according to:

$$
F_{i+\frac{1}{2}}^{S R}=\frac{1}{2}\left[F\left(\vec{q}_{L, i+\frac{1}{2}}^{S R}\right)_{i+\frac{1}{2}}+F\left(\vec{q}_{R, i+\frac{1}{2}}^{S R}\right)_{i+\frac{1}{2}}\right]
$$

This low-dissipation flux, while accurate in regions of smooth flow, is incapable of resolving shock discontinuities and therefore must be combined with a more conventional dissipative flux.

To construct the necessary dissipative flux, the piecewise-parabolic method of Collela and Woodward ${ }^{13}$ is first used to reconstruct the primitive state variables at the left and right sides of the cell interface. A minmod-type limiting procedure ${ }^{9}$ is used as a means of capturing discontinuities. With the higher-order states computed at the interfaces, the Harten, Lax, van Leer, and Contact (HLLC) scheme is then used to compute the dissipative flux. ${ }^{14,15}$ The HLLC scheme is a successor to the Harten, Lax, and van Leer (HLL) scheme and is designed to include treatment of the missing contact surface.

With the low-dissipation and dissipative fluxes computed, the final hybridized inviscid interface flux is computed according to:

$$
F_{i+\frac{1}{2}}^{H Y}=\Gamma_{S S} F_{i+\frac{1}{2}}^{U P}+\left(1-\Gamma_{S S}\right) F_{i+\frac{1}{2}}^{S R}
$$

where $\Gamma_{\mathrm{SS}}$ is a flow-dependent scaling factor and is referred to as a "shock sensor" due to its functional purpose. As briefly discussed before, application of LES to high-Mach number flows requires dissipative schemes for capturing shocks and low-dissipation schemes for resolving vortical motions. A shock sensing switch is thus used to dynamically adjust the solver for conditions near to and away from a shock. As illustrated above, the shock sensor is used to scale the respective flux components comprising the hybridized inviscid flux reconstruction. In this study, two methods for sensing shocks are used: the Ducros sensor ${ }^{16}$ and the approach of Larsson. ${ }^{17}$ The Ducros sensor is computed as:

$$
\Gamma_{S S}=(\nabla \cdot \vec{u})^{2} /(\nabla \cdot \vec{u})^{2}+(\nabla \times \vec{u})^{2}+
$$

where is a small number preventing a divide-by-zero operation when flow gradients are not present. The Larsson sensor is calculated according to: 


$$
\begin{gathered}
\Gamma_{S S}=\left\{\begin{array}{l}
0 \text { for } \theta \leq 1 \\
1 \text { for } \theta>1
\end{array}\right. \\
\theta=-(\nabla \cdot \vec{u}) /{ }_{\max }\left(L_{S S, 1}|\nabla \times \vec{u}|, L_{S S, 2} \frac{a}{d s}\right)
\end{gathered}
$$

where $a$ is the sound speed and $d s=\sqrt[3]{\text { cell volume }}$. The constants $\mathrm{L}_{\mathrm{ss}, 1}$ and $\mathrm{L}_{\mathrm{ss}, 2}$ were set to 5.0 and 0.05 , respectively, for the current study. Furthermore, the lower bound for each of these sensors was modified to provide a small level of background dissipation. Rather than allowing it to range from 0.0 to 1.0 , each shock sensor is bounded by 0.2 and 1.0, thereby preventing numerical instabilities arising within the shear layer reattachment region.

\section{B. Turbulence Recycling Procedure}

In order to introduce coherent turbulent structures at the inflow, turbulent fluctuations and relevant turbulence properties are recycled from a plane one cavity-depth upstream of the step. Turbulent fluctuations are extracted from the donor plane by subtracting time- and span-averaged mean flow data from the instantaneous flow data. These fluctuations are scaled according to boundary-layer similarity laws before being superimposed to a mean flow RAS profile. The general procedure used for this process is described by Edwards and others, ${ }^{9,18,19}$ and several important characteristics of the procedure are described here.

In order to prevent excessive turbulent energy accumulation in the outer regions of the boundary layer, an intermittency factor is applied to the recycled fluctuations. This intermittency factor is applied to density, temperature, and velocity components according to Eq. (9) and to turbulent kinetic energy, turbulence frequency, and the hybrid switching function according to Eq. (10).

$$
\begin{gathered}
q_{\text {inflow }}=q_{R A S}+F_{K l e b} q_{\text {recyc }}^{\prime} \text { for } q=\rho, T, u_{i} \\
q_{\text {inflow }}=\left(1-F_{K l e b}\right) q_{R A S}+F_{K l e b} q_{\text {recyc }} \text { for } q=k, \omega, \Gamma \\
F_{\text {Kleb }}=\left[1+\left(d / C_{\text {Kleb }} \delta\right)^{6}\right]^{-1}
\end{gathered}
$$

In the preceding equations, $q$ is a placeholder for the variables referenced above. The intermittency factor, $\mathrm{F}_{\mathrm{kleb}}$, is a Klebanoff-type intermittency function, ${ }^{20}$ where $\mathrm{C}_{\text {kleb }}=1.10$. This modification ensures that the RAS free stream inflow properties are not modified outside the boundary layer by the recycling procedure.

A further modification of the general recycling procedure is to consider pressure fluctuations as they relate to the calculation of density fluctuations. Following the Morkovin hypothesis ${ }^{20}$ which assumes that total temperature fluctuations are negligible, the temperature fluctuations are related to the velocity fluctuations. The recycled temperature fluctuations are then limited to prevent their magnitude from exceeding that predicted by Morkovin's hypothesis. Using these limited temperature fluctuations, a provisional pressure fluctuation is calculated, which is also limited according to some user-defined multiple of the mean static pressure (chosen as 0.02 in the present simulations). The limited recycled temperature and pressure fluctuations are used in conjunction with the mean temperature and pressure to compute corrected density fluctuations.

\section{Computational Execution}

Simulations are performed on a structured three-dimensional grid consisting of approximately 35 million cells for the fine level and approximately 8.8 million cells for the coarsened level. The coarse grid was constructed by coarsening the fine grid once in each of the streamwise and spanwise directions, where coarsening is defined as removing every other grid point in a given direction. The grid is composed of four primary blocks, as shown in Fig. 3. The two upper blocks are connected to the lower blocks via non-C0 continuous patches. Similarly, the lower left block is attached to the lower right block by a non- $\mathrm{C} 0$ continuous patch where only the spanwise coordinates are mismatched (the downstream spacing is twice that of the upstream spacing). For the fine grid, the I-J-K 
(streamwise-transverse-spanwise or $\mathrm{x}-\mathrm{y}-\mathrm{z}$ ) dimensions of the blocks adjacent to the wall directly upstream and downstream of the step are $525 \times 121 \times 301$ and $453 \times 241 \times 151$, respectively; dimensions of the upper left and right blocks are $29 \times 45 \times 15$ and $153 \times 45 \times 15$, respectively. The patches connecting the lower and upper blocks are positioned far enough from the domain of interest to ensure that resolved boundary layer and free shear layer eddies are not convected into them. The physical dimensions in the $\mathrm{x}, \mathrm{y}$, and $\mathrm{z}$ directions of the computational domain are $28.6 \mathrm{~cm}, 22.5 \mathrm{~cm}$, and $3.81 \mathrm{~cm}$, respectively. The flat plate extended four step heights upstream of the step, which is a factor of two further upstream than in previous studies ${ }^{5,21}$ to allow for recycling of turbulent fluctuations, and the grid was clustered to provide a nominal $y^{+}$of 1.0 adjacent to the surface. The streamwise and spanwise grid spacing was set to $1 / 20^{\text {th }}$ of the boundary layer thickness. Downstream of the step, the grid clustering required by the approach flow boundary layer was relaxed toward a more isotropic grid for the resulting shear layer. The maximum $\mathrm{x}, \mathrm{y}$, and $\mathrm{z}$ spacing in this region was $2.5 \%, 5.0 \%$, and $1.0 \%$, respectively, of the cavity depth $(2.54 \mathrm{~cm})$. The cell aspect ratio for the LES regions was no greater than approximately 20.0. The three-dimensional grid was split into 1980 sub-blocks to allow runtime parallelization using Message Passing Interface (MPI) on NASA Advanced Supercomputing resources. Each simulation performed on the fine grid required approximately 240 hours of wall clock time on 512 processors. The same simulations performed on the coarse grid required about 60 hours of wall clock time on 512 processors.

Inflow variable profiles and flowfield initializations for the LES/RAS cases were obtained using the converged RAS results of case NR8RAS. For this case, the Menter $k$ - $\omega$ turbulence model ${ }^{7}$ was used with a compressibility correction $^{22}$, and the free stream flow was allowed to develop until the momentum thickness one cavity-depth upstream of the step matched experimental measurements. A significant spread in solutions was obtained using various turbulence models for the preliminary RAS simulation, and the RAS results used in this study represent those in best agreement with experimental data. Nominal inflow conditions included: static pressure of $21.2 \mathrm{kPa}$, Mach number of 2.92, and temperature of $92.4 \mathrm{~K}$. For all LES/RAS cases, homogeneous turbulence was assumed in the spanwise direction allowing the use of periodic boundary conditions at both boundaries. For the fine grid cases, all walls were assumed adiabatic and no slip. For the coarse grid cases, all walls except for the vertical cavity wall were assumed adiabatic and no slip; wall functions were applied at the vertical cavity wall due to excessively high $y+$ values observed there during runtime. For all runs, an extrapolation condition was applied at the exit plane, and a characteristic condition was enforced at the far-field plane. The inflow profiles obtained using case NR8RAS were applied at the inflow plane for each subsequent case. For cases in which turbulence recycling was enabled, turbulent fluctuations were superimposed onto the initial RAS solution upstream of the cavity step. These turbulent fluctuations were extracted from a previously-simulated fully-resolved (via LES) turbulent boundary layer on a flat plate. This initialization procedure provides the turbulence necessary for effective recycling without necessitating the natural development of such structures.

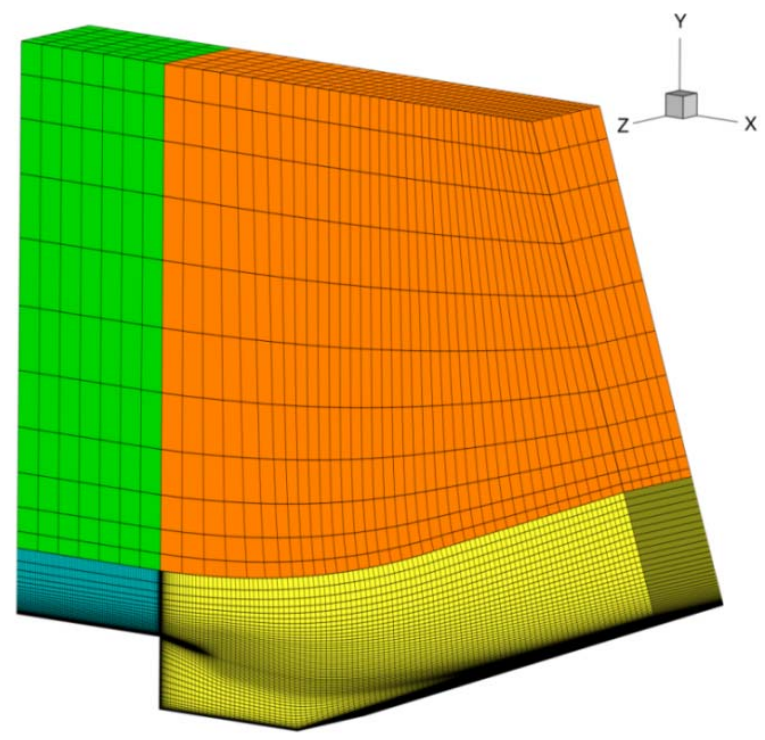

Figure 3. Isometric view of the three-dimensional domain coarsened twice in the streamwise and transverse directions and once in the spanwise dimension for visual clarity and to emphasis grid design. 
The governing equations were integrated using a dual-time-stepping approach ${ }^{23}$ in which the diagonalized approximate factorization (DAF) method ${ }^{24}$ is used for integration in pseudo time with a maximum CourantFriedrichs-Lewy (CFL) number of 100.0, and a second-order three-point backwards finite difference approximation was used for integration in physical time. Convergence was defined as achieving a residual reduction of 2.5 ordersof-magnitude or as reaching a limiting number of 15 sub-iterations. The physical time step was set to $0.1 \mu \mathrm{s}$. Following initialization, the flowfield was evolved for a minimum of 10 characteristic flow-through times before taking statistics. A characteristic flow-through time was defined as the time it takes a particle in the free stream to travel from the corner of the step to the point of reattachment of the free shear layer along the inclined wallapproximately $0.2 \mathrm{~ms}$. Once a statistically stationary state was achieved, flowfield properties were ensembleaveraged in time at a rate of $0.5 \mu$ s for periods of 5, 10, and 15 flow-through times and subsequently averaged spatially in the homogeneous spanwise direction. The RAS turbulence model constants were set according to Ref. 7, and SGS turbulence model constants were set as suggested in Ref. 8.

\section{Results and Discussion}

Fifteen characteristic flow-through times were necessary to achieve reliable statistics. As illustrated in Fig. 4, the simulations reproduced the major flow phenomena observed in the experiment; the supersonic flow upstream of the cavity detaches at the step corner, thereby creating a free shear layer over the cavity. This free shear layer attaches along the inclined wall and interacts with an oblique shock front standing off the inclined wall. Fluid is entrained into the cavity near the location of shear layer attachment, and this fluid creates a recirculation zone which drives an unsteady low-frequency motion of the free shear layer. Note that in Fig. 4, annotations are included that indicate major flow features and identify each image's relative location in time, which is given as the value of elapsed time relative to an arbitrary origin, $\Delta \mathrm{t}$, divided by the characteristic flow-through time, $\Delta \tau$. In the following sections, results are presented and organized according to study objective, including: assessment of solution accuracy via comparison to experiment, assessment of grid sensitivity, isolation of inflow turbulence recycling effect, and determination of shock sensor effect on solution accuracy.

\section{A. Comparison with Experiment}

Before comparing results to experimental data, the boundary layer was confirmed to have developed properly upstream of the step. This confirmation was necessary to ensure that the constant, $\alpha$, required for the recycling procedure was chosen appropriately and that the recycling procedure itself did not adversely affect the inflow boundary layer. For both the fine grid and coarse grid cases, boundary layer velocity profiles at several planes upstream of the step were compared to theoretical predictions based on Cole's Law-of-the-Wall formulation and the original RAS solutions under the van Driest transformation described in Ref. 9. These three planes included the inflow plane, the donor recycling plane, and a plane at the step corner. For each case and at each plane, the averaged LES/RAS results agreed well with both the theoretical log-layer predictions and with RAS data. The comparisons for cases R35D and R8D at the recycling plane are included in Fig. 5 to demonstrate the level of agreement. In each case and at each plane, the LES/RAS simulations reproduced the slope and location of the loglayer well, and the blending function anchored the mean LES/RAS transition point near the upper edge of the loglayer. Thus, the LES/RAS approach and recycling procedure provide proper development of the boundary layer upstream of the step. 

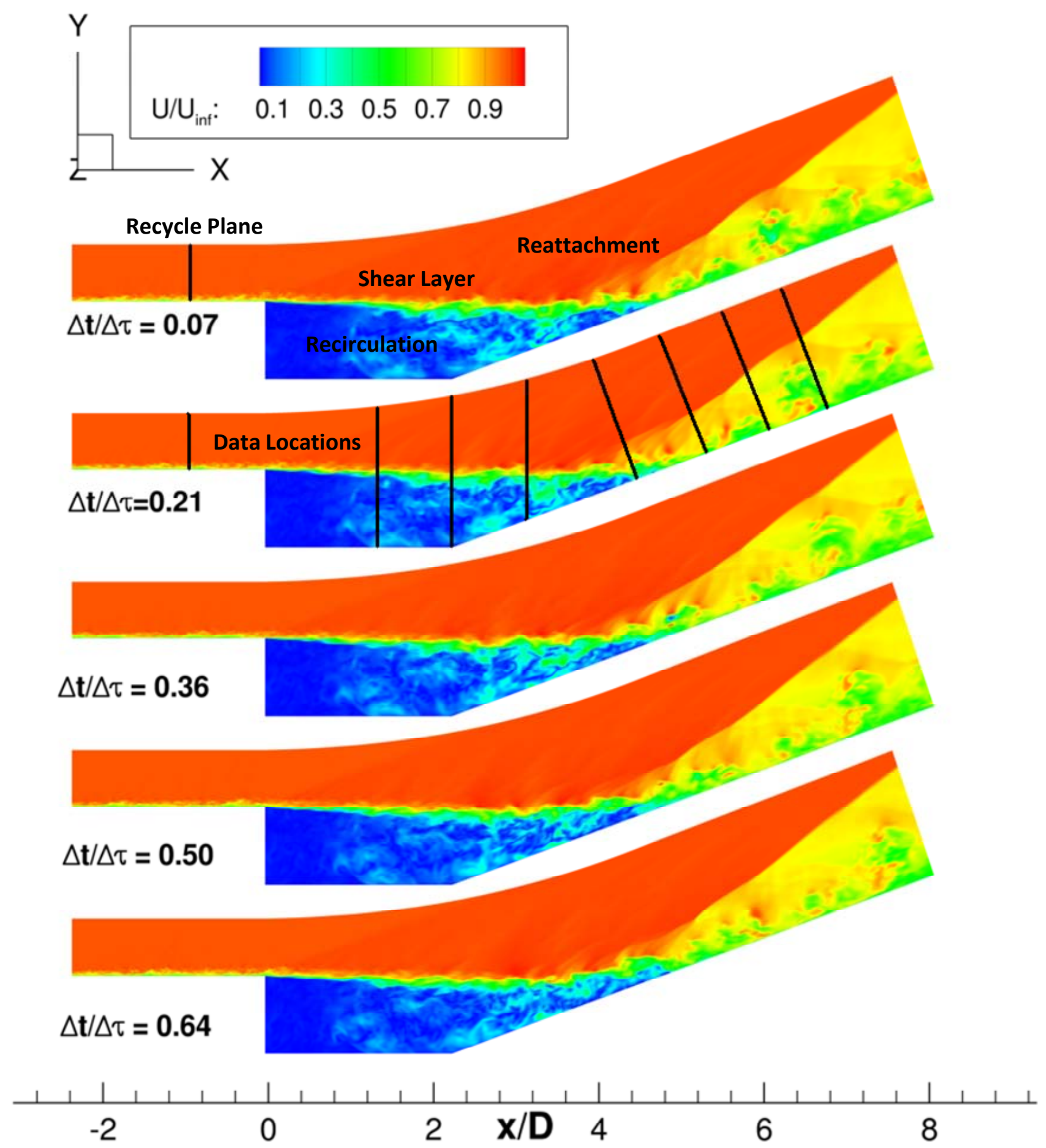

Figure 4. Instantaneous snapshots of velocity magnitude at the spanwise two-dimensional centerline plane for case R35D with recycle plane, major flow features, and data extraction locations marked. 


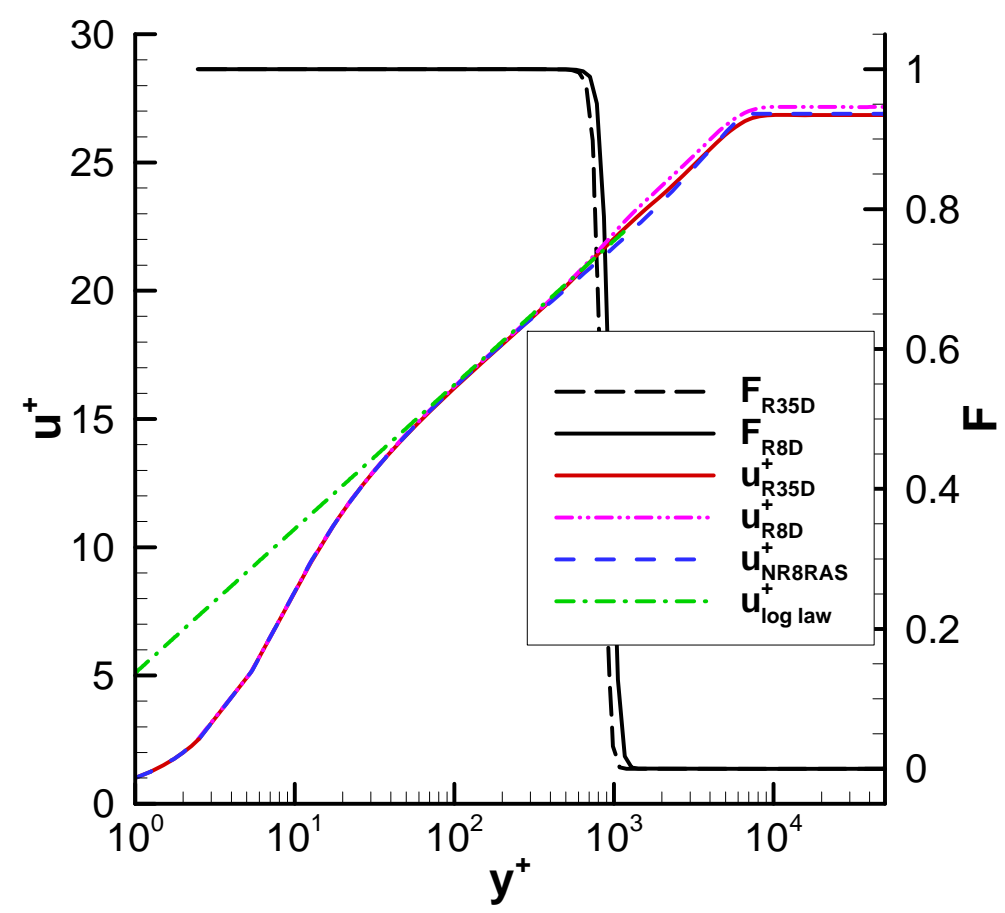

Figure 5. Comparisons of velocity and blending function through the approach boundary layer for cases $R 35 D$ and $R 8 D$ at the recycling plane.

With the upstream boundary layer validated against theoretical predictions, downstream data obtained from the simulations are compared with that of experiments. Case R35D - the fine grid case using the Ducros sensor-is designated the baseline case. In Figs. 6a through $6 \mathrm{~d}$, velocity and pressure results are compared to available experimental data and to the compressibility-corrected RAS data for case NR8RAS. Note that a variable having a superscripted * indicates a value normal to a solid surface, and all spatial dimensions are normalized by the cavity depth, D. Overall, the LES/RAS results agree quite well with the experimental data after shear layer reattachment, and a mismatch is noted in the shear layer profiles. The deviation apparent in these results is an under-prediction of the vertical location of the shear layer, which is most-clearly visible in the shear layer velocity profiles. This deficiency also appears in the velocity and pressure profiles, normalized by free stream values, normal to the inclined wall near the point of reattachment $\left(x^{*} / D=2.7\right)$. The assumption of turbulent homogeneity in the spanwisedirection may be the cause for this issue, since previous efforts have shown that three-dimensional effects play a significant role in flow separation even when extensive steps are taken in the experiments to ensure some level of two-dimensionality. ${ }^{25}$ In this experiment, flow fences were placed on either side of the compression ramp. However, the interaction of the reattaching shear layer with theses fences was not captured in the current simulations. It is also plausible that the width of the homogeneous direction was not large enough to adequately capture the largest scales associated with the recirculation region within the cavity. A future effort is planned to assess this possibility. Predictions of the normalized static pressure along the inclined wall are considerably better for LES/RAS, as well as predictions of the normalized velocity and pressure profiles at planes extending normal to the inclined wall. This result was expected given the fact that RAS models tend to severely over-predict the boundary layer recovery rate when free shear flows reattach to solid surfaces. 
$x / D=-1.0 \quad x / D=1.5 \quad x / D=2.5 \quad x / D=3.5$

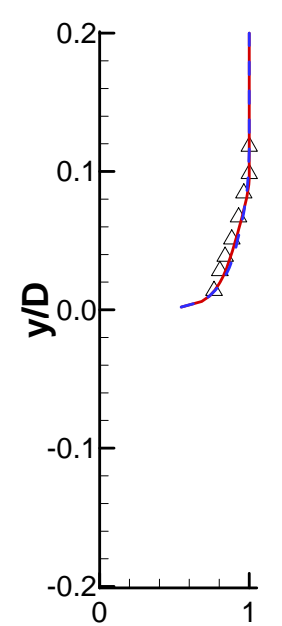

a)

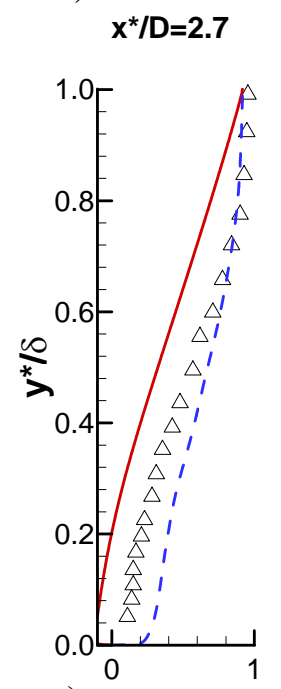

c)

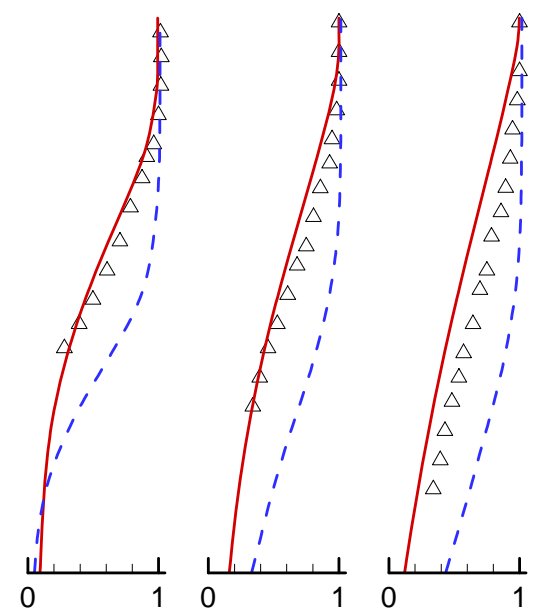

u/U $\mathbf{U}_{\infty}$ $x^{*} / D=3.8$

$\mathrm{X}^{\star} / \mathrm{D}=4.6$

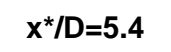

$x^{*} / D=5.4$

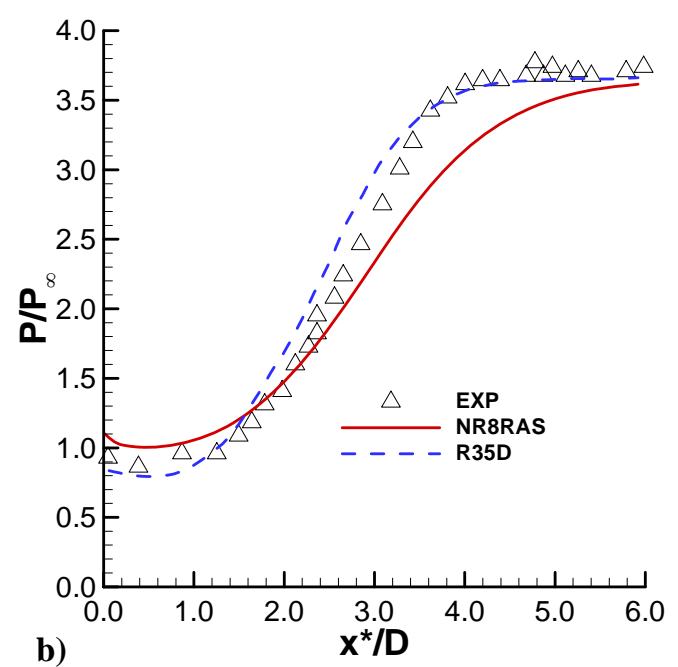

$x^{*} / D=2.7 \quad x^{*} / D=3.8$

$x^{*} / D=4.6 \quad x^{*} / D=5.4$

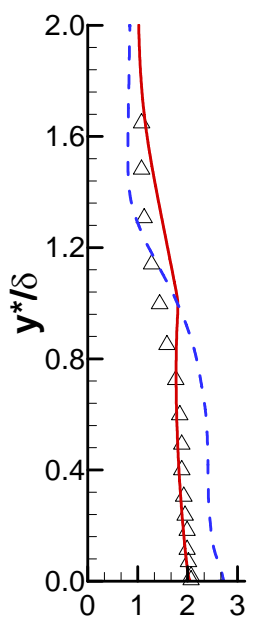

d)

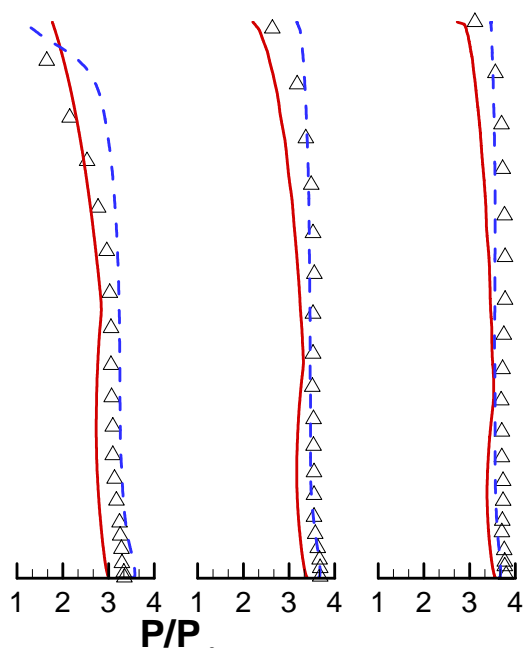

$\mathbf{P} / \mathbf{P}_{\infty}$

Figure 6. Comparisons of normalized velocity and pressure for cases R35D (blue dashed line) and NR8RAS (solid red line) to experiment (open triangles).

Though the vertical location of the shear layer is under-predicted, the shear layer spreading rate is captured quite accurately by the LES/RAS data. This can be seen by translating the velocity curves through the shear layer vertically until the uppermost point of inflection of the simulation data intercepts the experimental data. Figure 7 shows these vertically-translated curves, which demonstrate the difference in spreading rate predictions between RAS and LES/RAS. Assuming the shear layer forms a line across the cavity, the simulated shear layer for the baseline case is deflected about $1.8^{\circ}$ downward from the experimental data. The good spreading rate agreement, when considered in light of the mismatch in vertical displacement of the shear layer, strengthens the previous discussions on the design differences between the experiment and simulations. Not only is it likely that the experiment's aerodynamic fences only partially alleviate three-dimensional effects, but the limited physical extent of the computational domain's spanwise direction may cause further deviation from experiment due to artificial limiting of the size of the largest spanwise flow features that can reside within the recirculation region. 


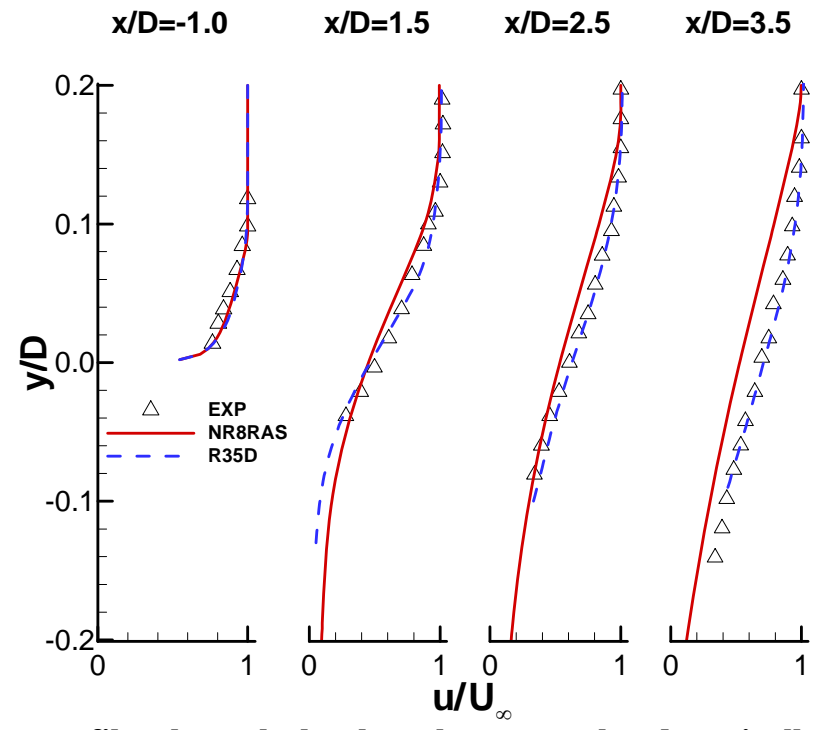

Figure 7. Normalized velocity profiles through the shear layer translated vertically to demonstrate spreading rate agreement for cases R35D (blue dashed line) and NR8RAS (red solid line), as compared to experimental data (open triangles).

\section{B. Grid Sensitivity}

An assessment of the grid sensitivity was made by comparing results of cases R35D and R8D, as the only difference between these runs was the number of grid cells used to represent the physical domain. The grid used for case R8D was identical to that of case R35D, albeit coarsened once in the streamwise and spanwise directions. The transverse direction was not coarsened because the resulting grid would have given $y+$ values too large for integrateto-the-wall simulations. Each case was evolved in time for ten flow-through times before statistics were collected for 5,10 , and 15 flow-through times. The grid sensitivity of the solution was found to be significant. The coarsened grid is unable to resolve adequate levels of turbulence, as compared to the fine grid. The level of resolved turbulence captured by each grid is significantly different, as illustrated in Fig. 8, where instantaneous Mach number contours are presented at a two-dimensional slice at the spanwise centerline. Note that while the turbulent fluctuations in the approach flow boundary layer are well-resolved for both cases, these fluctuations and corresponding turbulent motions are resolved to a lesser degree moving downstream of the step when the coarse grid is utilized. This observation is further illustrated through examination of the contributions of resolved TKE and modeled TKE downstream of the step and is demonstrated in Fig. 9, where line plots of the TKE contributions are presented at several planes. At each of these planes, a measure of the resolved turbulent content is approximated according to:

$$
\% T K E_{\text {res }}=\frac{T K E_{\text {res }}}{T K E_{S G S}+T K E_{\text {res }}} \times 100
$$

Calculations using Eq. (12) are performed using peak-to-peak values from Fig. 9. Whereas in case R35D, the turbulence is $71 \%, 80 \%$, and $85 \%$ resolved at each plane, respectively, the same is not true for case R8D. For case R8D, the turbulence is only $30 \%, 36 \%$, and $50 \%$ resolved at each of the three planes, respectively. Typically for LES of high Reynolds number flows, the percentage of resolved turbulence is in the $80-90 \%$ range. ${ }^{26,27,28}$ The dominance of modeled TKE leads to more reliance on the SGS model and, in this case, a larger disagreement with the available experimental data. This disagreement is highlighted in Fig. 10, where the results of case R8D are plotted with the results of case R35D and the experimental data through the shear layer. Note that the data for each case have been shifted, as described earlier, to highlight the difference in spreading rates. 

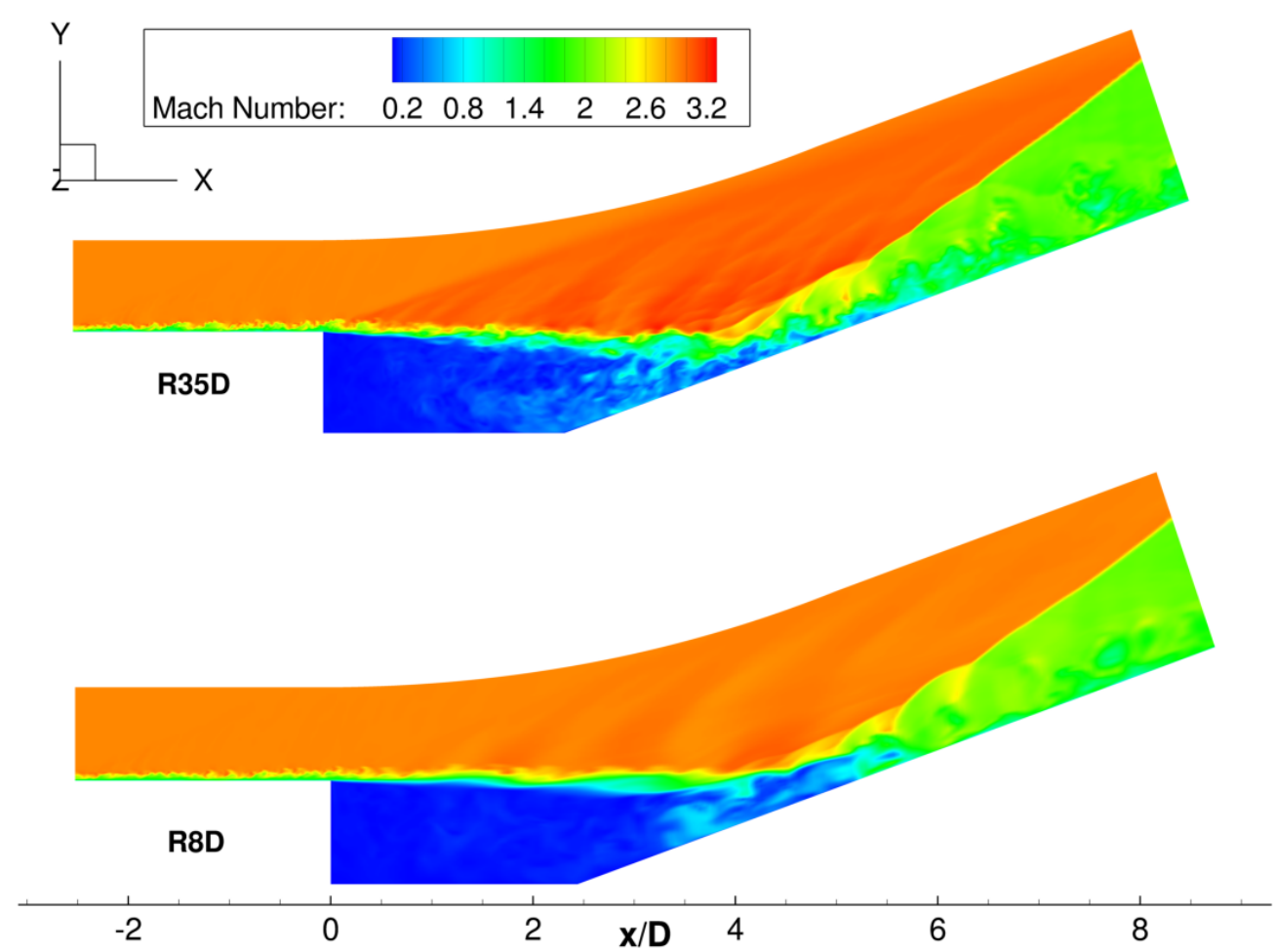

Figure 8. Instantaneous Mach number snapshots for cases R35D (top) and R8D (bottom) at the spanwise centerline plane.
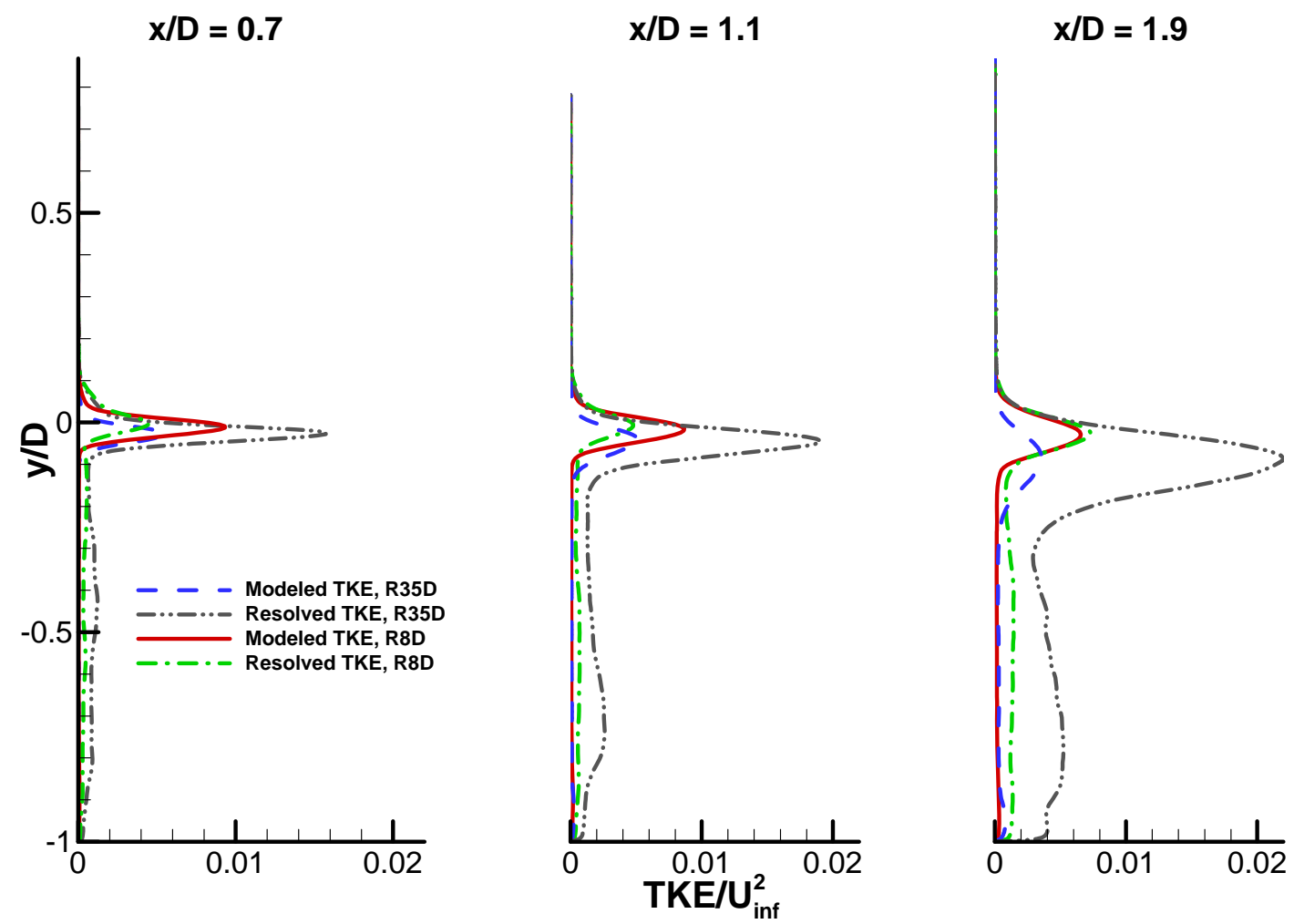

Figure 9. TKE contributions at three planes downstream of the step for cases R35D and R8D. The blue dashed line and red solid line represent the modeled TKE for cases R35D and R8D, respectively, and the gray dash-dot-dot line and green gash-dot line represent the resolved TKE for cases R35D and R8D, respectively. 


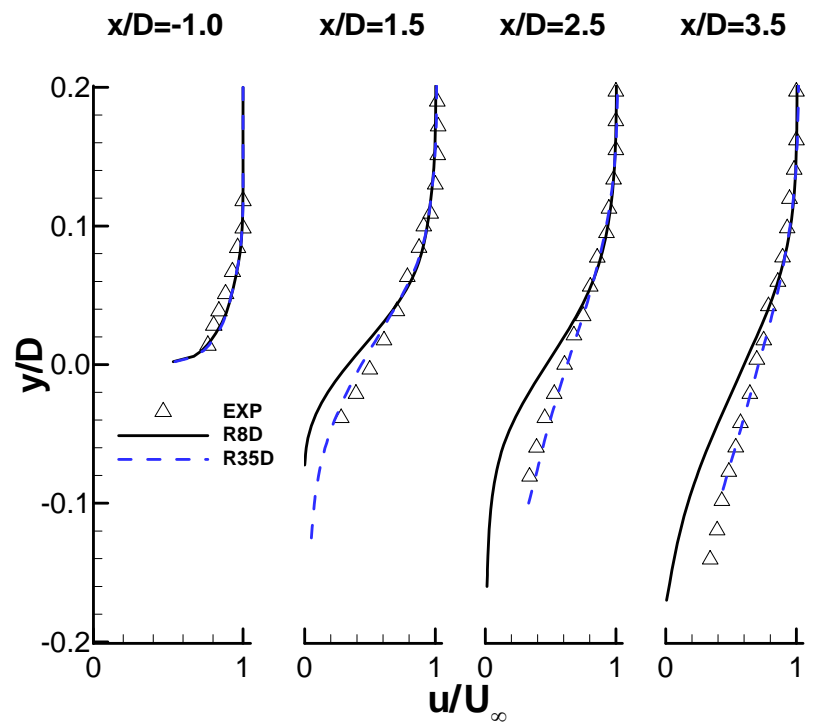

Figure 10. Normalized velocity results through the shear layer translated vertically for cases R35D (blue dashed line) and R8D (black solid line), as compared to experiment (open triangles).

\section{Effect of Inflow Turbulence Recycling}

The effect of inflow turbulence recycling on the flowfield was isolated by comparing the results of cases R8D and NR8D. For these two cases, the only differentiating factor is the inflow turbulence recycling, which is enabled in the first and disabled in the second case, respectively. Specifically, for case NR8D, the RAS solution from case NR8RAS was used to specify the inflow conditions at the corner of the step; the LES/RAS governing equations were then solved in space and time for the flowfield downstream of the step. This treatment is similar to how a detached eddy simulation approach would proceed, where attached boundary layer regions are treated as pure RAS and only detached regions are treated with LES. Accounting for resolved fluctuations in the approach boundary layer had little effect on the shear layer development as demonstrated by the results of cases R8D and NR8D in Fig. 11. The shifted velocity profiles through the shear layer are in relatively good agreement between the two cases. Keeping in mind that the grid used in cases R8D and NR8D inadequately resolved turbulence downstream of the step, the current results indicate the inflow turbulence recycling had little effect on the shear layer development for the current flow.

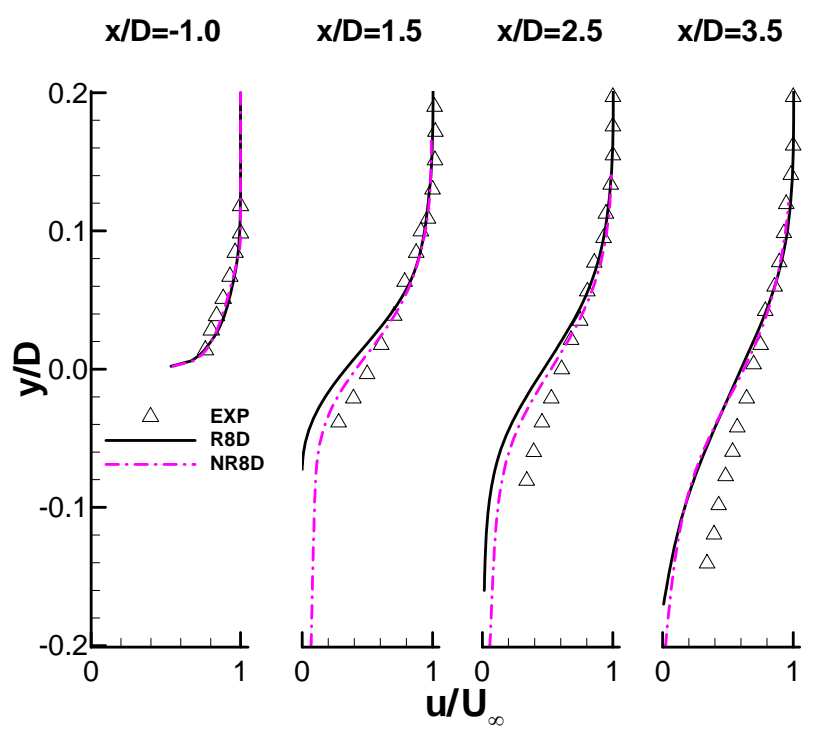

Figure 11. Normalized velocity results through the shear layer for cases R8D (black solid line) and NR8D (magenta short-dash line), as compared to experiment (open triangles). 


\section{Effect of Shock Sensor}

In order to determine the effect that choice of shock sensor may have on the solution, cases R35D and R8D were repeated using the Larsson shock sensor in place of the Ducros sensor. The Ducros sensor was considered the baseline shock sensor for purposes of this work. In their implementation, these shock sensors compare the magnitude of the divergence and curl of velocity; however, the Larsson sensor uses a slightly different formulation, which includes logic that toggles the interface flux approximation to either fully-upwind or fully-symmetric. Conversely, the Ducros switch blends the two independent interface flux approximations in proportion to the magnitude of the normalized sensor coefficient.

Instantaneous snapshots of the shock sensor for cases R8D and R8L are presented in Fig. 12. Note that the magnitude of the shock sensor differs significantly across the flowfield between the two cases. As explained previously, the Ducros sensor takes on values between the upper and lower bounds of the switch throughout most of the domain, unlike the Larsson sensor, which forms more distinct regions limited to the upper and lower sensor bounds. Though the sensor distributions are substantially different, the results of cases R35D and R35L, and of cases R8D and R8L, are in relatively good agreement. Normalized velocity and pressure profiles, through the shear layer and along the inclined wall, are presented in Fig. 13 for cases R35D and R35L. The velocity profiles in Fig. $15 \mathrm{a}$ have been shifted to compare shear layer spreading rate and illustrate good agreement. Assuming a line connects the curve at $x / D=3.5$ to the corner of the step, the angular deviation between the two shear layers is about $0.85^{\circ}$. The pressure profiles plotted in Fig. $15 \mathrm{~b}$ exhibit a small difference, likely attributed to the vertical displacement of the shear layer. In case R35L, the shear layer intersects the vertical wall slightly lower than in case $\mathrm{R} 35 \mathrm{D}$, and this causes a strengthened oblique shock off the inclined wall. This drives a higher rise in pressure along the inclined wall and alters the velocity and pressure profiles normal to the inclined wall at the first data station, $x * / D=2.7$. Comparisons of cases R8D and R8L indicate even better agreement than those included in Fig. 15 and are therefore omitted here. Based on these comparisons, it is reasonable to conclude that the choice of shock sensor has little effect on the statistics for this flowfield.
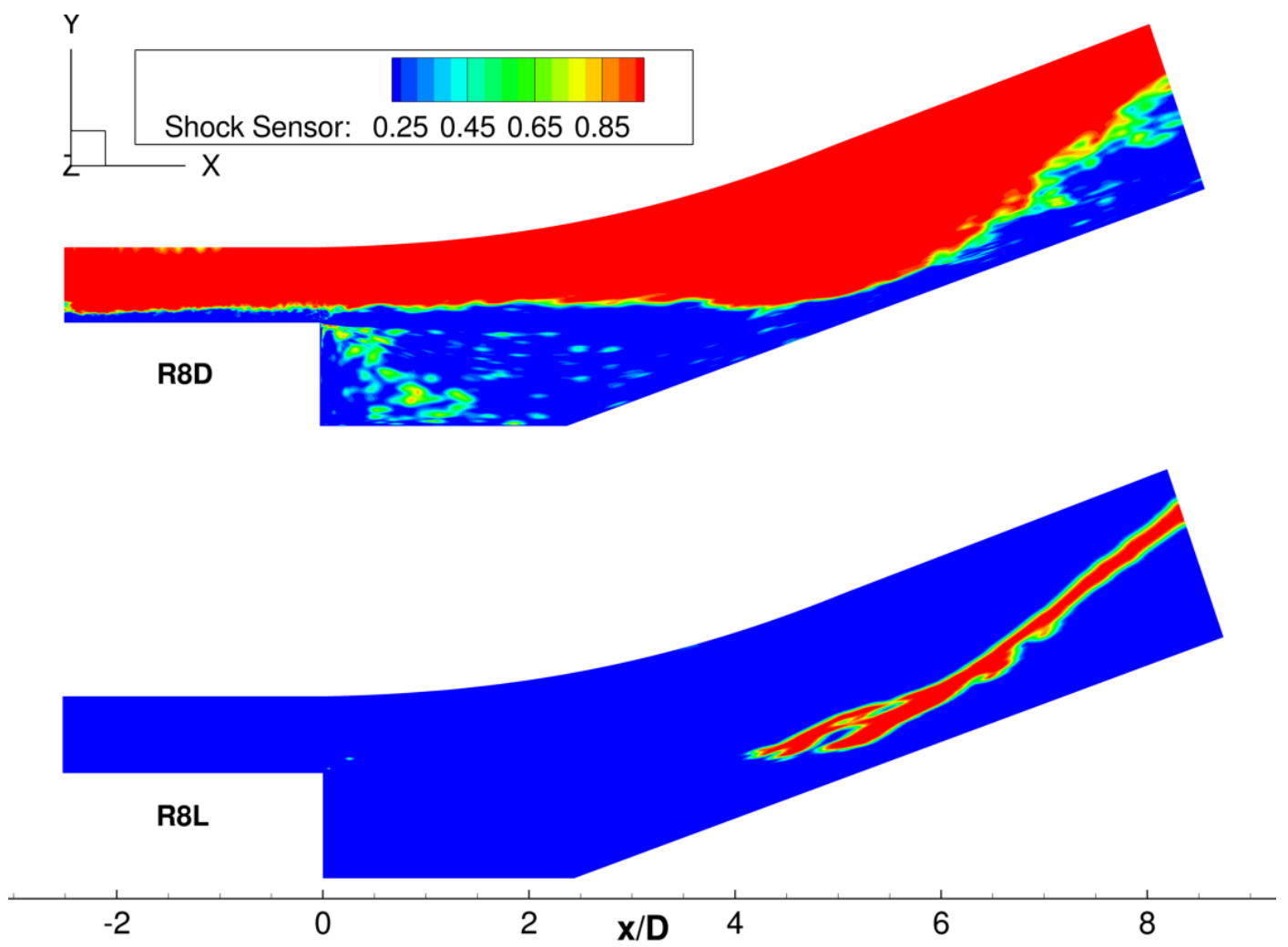

Figure 12. Instantaneous shock sensor contours for cases R8D (top) and R8L (bottom) at the spanwise centerline plane. 

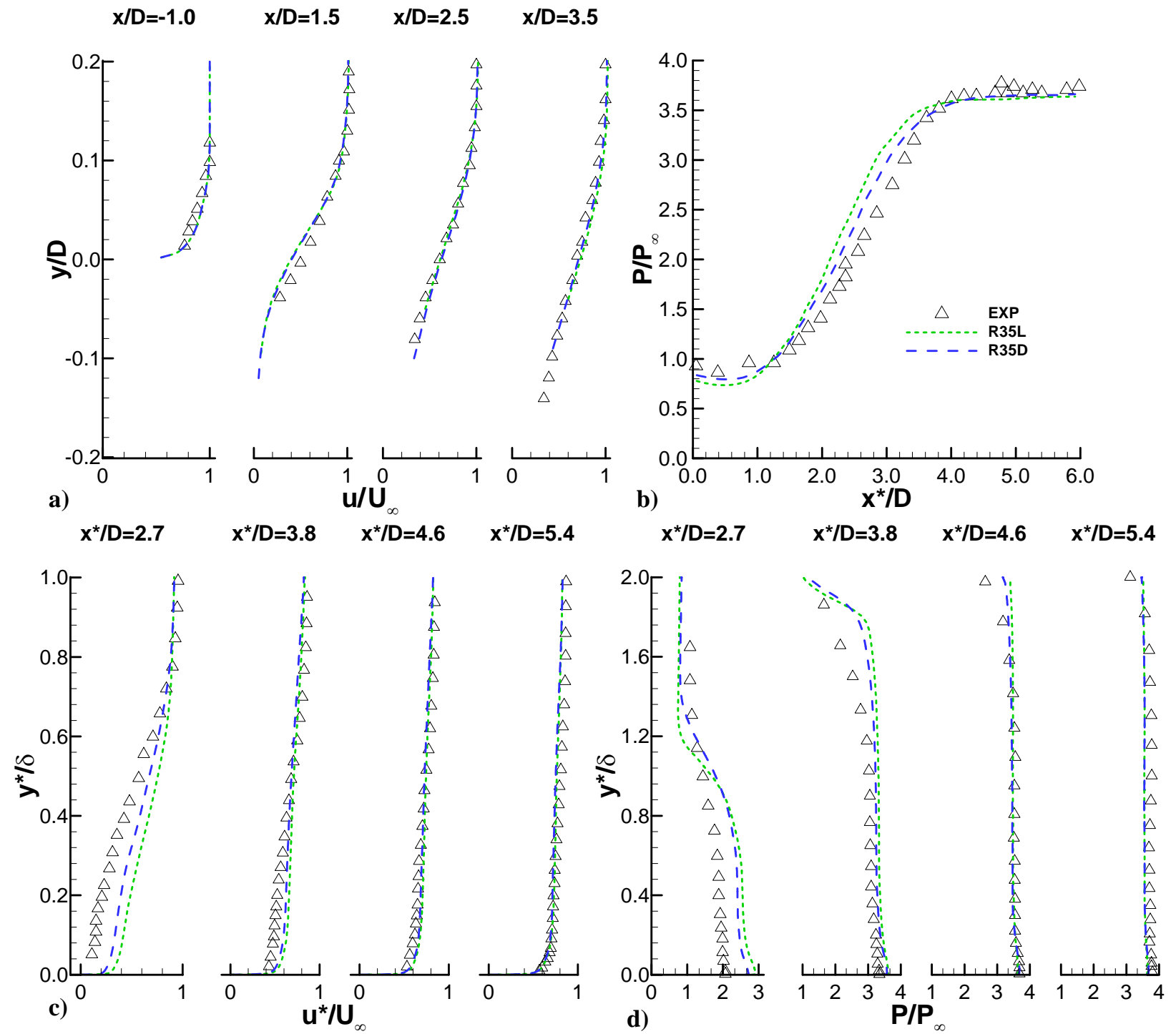

Figure 13. Comparisons of normalized velocity and pressure for cases R35D (blue long-dash line) and R35L (green short-dash line) to experiment (open triangles).

\section{Summary and Conclusions}

Several simulations were performed of a supersonic cavity flow exhibiting a reattaching free shear layer utilizing recent developments to the VULCAN CFD code. The simulations were designed in such a way as to: assess the applicability of LES/RAS to the chosen cavity flow, isolate the effect of inflow turbulence recycling on the flowfield statistics, determine any sensitivity of the solution to grid density, and evaluate the effect of shock sensor choice in the use of a hybridized flux reconstruction scheme. Two grids were used-a fine grid consisting of 35 million cells and a coarsened version of the same grid constructed of 8.8 million cells. A baseline run was performed on the fine grid using the Ducros sensor. Two cases were performed on the coarsened grid toggling the inflow turbulence recycling on and off in conjunction with the Ducros switch. The case performed on the coarse grid with recycling enabled was repeated using the Larsson switch to isolate the effect of shock sensor choice.

Results that utilized the baseline fine grid compared quite well with experimental data outside of the cavity region. Through the cavity region, velocity profiles indicated good shear layer spreading rate agreement but a deviation in vertical location of the shear layer. This disagreement may be a result of the assumption of turbulent homogeneity in the spanwise z-direction or an insufficient spanwise width that could limit the size of spanwise structures. Even though measures were taken during the experiment to promote two-dimensionality, such as aerodynamic fences, previous studies have indicated that such approaches likely do not completely alleviate the 
effects of three-dimensionality. For this case, it is easy to imagine three-dimensional motions arising from fluidic interactions with the corners of the finite-width cavity and with the fences. These same three-dimensional motions could potentially alter the dynamics of the shear layer. In future studies of this flow, three-dimensional effects should be considered to better capture these effects.

Simulation results suggest that the solution is strongly grid-sensitive given the level of resolution utilized here. Hence, it cannot be confirmed that the finest grid employed is providing an acceptable level of grid convergence. However, simulations performed on the fine grid proved to resolve a large fraction of the turbulence, whereas the coarse grid simulations exhibited dominance of the subgrid modeled turbulence. Further, the coarse grid results suggest the inflow turbulence recycling plays a small role in the free shear layer development. Lastly, when used with the hybridized inviscid flux reconstruction method, the choice of shock sensor has little effect on the resulting flowfield. The sensors of Ducros and Larsson were considered for this work, and the results illustrate the relative insensitivity to the detailed functional form chosen for the shock sensor.

\section{Acknowledgments}

This work was supported primarily by a NASA Space Technology Research Fellowship (training grant NNX11AM86H), with additional support provided by the Virginia Space Grant Consortium and by a Virginia Commonwealth Fellowship. The authors are grateful for the helpful discussions with Dr. Tomasz Drozda during the course of this study. Computational resources were provided by the NASA Advanced Supercomputing Division.

\section{References}

${ }^{1}$ Settles, G.S., Baca, B.K., Williams, D.R., and Bogdanoff, S.M., "A study of reattachment of a free shear layer in compressible turbulent flow," AIAA Paper 80-1408, July 1980.

${ }^{2}$ Horstman, C.C, Settles, G.S., Bogdanoff, S.M., and Williams, D.R., "A reattaching free shear layer in compressible turbulent flow-A comparison of numerical and experimental results," AIAA Paper 81-0333, Jan. 1981.

${ }^{3}$ Settles, G.S., Williams, D.R., Baca, B.K., and Bogdanoff, S.M., "Reattachment of a compressible turbulent free shear layer," AIAA J., Vol. 20(1), 1982, pp. 60-68.

4,'Viscous Upwind Algorithm for Complex Flow Analysis (VULCAN): User Manual," Online, 2010.

${ }^{5}$ Baurle, R.A., Tam, C.J., Edwards, J.R., and Hassan, H.A., "A hybrid simulation approach for cavity flows: blending, algorithm, and boundary treatment issues," AIAA J., Vol. 41(8), 2003, pp. 1463-1480.

${ }^{6}$ Baurle, R.A. and Edwards, J.R., "Hybrid Reynolds-averaged/large-eddy simulations of a coaxial supersonic freejet experiment," AIAA J., Vol. 48(3), 2010, pp. 551-571.

${ }^{7}$ Menter, F.R., "Zonal two equation k- $\omega$ models for aerodynamic flows," AIAA Paper 92-0439, Jan. 1992.

${ }^{8}$ Yoshizawa, A., and Horiuti, K., "A statistically-derived subgrid scale kinetic energy model for large-eddy simulation of turbulent flows," J. Phys. Soc. of Japan, Vol. 54, 1985, pp. 2834.

${ }^{9}$ Choi, J.-I., Edwards, J.R., and Baurle, R.A., "Compressible boundary layer predictions at high Reynolds number using hybrid LES/RANS methods," AIAA Paper 2008-4175, June 2008.

${ }^{10}$ Edwards, J.R., Choi, J.-I., and Boles, J.A., "Hybrid LES/RANS simulation of a mach 5 compression-corner interaction," AIAA J., Vol. 46(4), 2008, pp. 977-991.

${ }^{11}$ Walz, A., Boundary Layers of Flow and Temperature, MIT Press, Cambridge, MA, 1969 (English translation).

${ }^{12}$ Gieseking, D., Choi, J-I., Edwards, J., Hassan, H., "Simulation of shock/boundary layer interactions using improved LES/RANS models," AIAA Paper 2010-111, Jan. 2010.

${ }^{13}$ Colella, P., and Woodward, P.R., "The piecewise parabolic method (PPM) for gas-dynamical simulations," J. Comp. Phys., Vol. 54, 1984, pp. 174-201.

${ }^{14}$ Batten, P., Clarke, N., Lambert, C., and Causon, D.M., "On the choice of wave speeds for the HLLC Riemann Solver," SIAM J. Sci. Comp., Vol. 18(6), 1997, pp. 1553-1570.

${ }^{15}$ Toro, E.F., Spruce, M., and Speares, W., "Restoration of the contact surface in the HLL-Riemann solver," Shock Waves, Vol. 4(1), 1994, pp. 25-34.

${ }^{16}$ Ducros, R., Ferrand, V., Nicoud, F., Weber, C., Darracq, D., Gacherieu, C., and Poinsot, T., "Large-eddy simulation of shock-turbulence interaction," J. Comp. Phys., Vol. 152(2), 1999, pp. 517-549.

${ }^{17}$ Larsson, J., Vicquelin, R., and Bermejo-Moreno, I., "Large-eddy simulations of the HyShot II scramjet," Center for Turbulence Research Annual Research Report Briefs, 2011.

${ }^{18}$ Xiao, X., Edwards, J.R., Hassan, H.A., and Baurle, R.A., "Inflow boundary conditions for hybrid large-eddy/Reynoldsaveraged Navier-Stokes simulations," AIAA J., Vol. 41(8), 2003, pp. 1481-1490.

${ }^{19}$ Fan, T.C., Edwards, J.R., Hassan, H.A., and Baurle, R.A., "Hybrid large-eddy/Reynolds-averaged Navier-Stokes simulations of shock separated flows," J. of Spacecraft and Rockets, Vol. 41(6), 2004, pp. 897-906.

${ }^{20}$ Smits, A.J., and Dussauge, J.-P., Turbulent shear layers in supersonic flow, $2^{\text {nd }}$ ed., Spring, New York, 2006.

${ }^{21}$ Fan, T.C., Xiao, X., Edwards, J.R., Hassan, H.A., and Baurle, R.A., "Hybrid RANS/LES simulation of a shock wave/boundary layer interaction," AIAA Paper 2002-0431, Jan. 2002.

${ }^{22}$ Wilcox, D.C., Turbulence Modeling for CFD, $1^{\text {st }}$ ed., DCW Industries, Inc., 1993. 
${ }^{23}$ Rai, M.M., "Navier-Stokes simulations of blade vortex interaction using high-order accurate upwind schemes," AIAA Paper 87-0543, 1987.

${ }^{24}$ Pulliam, T.H., "A diagonal form of an implicit approximate-factorization algorithm,” J. Comp. Phys., Vol. 39(2), 1981, pp. 347-363.

${ }^{25}$ Oliver, A.B., Lillard, R.P., Schwing, A.M., Blaisdell, G.A., and Lyrintzis, A.S., "Assessment of turbulent shock-boundary layer interaction computations using the OVERFLOW code," AIAA Paper 2007-0104, Jan. 2007.

${ }^{26}$ Pope, S.B., "Ten questions concerning the large-eddy simulation of turbulent flows," New J. Phys., Vol. 6(1), 2004.

${ }^{27}$ Sagaut, P., Large-eddy simulations of incompressible flows: an introduction, Springer, Berlin, Germany, 2006.

${ }^{28}$ Pope, S.B., Turbulent Flows, Cambridge University Press, New York, NY, 2000. 